\title{
A REACTION-DIFFUSION MODEL OF STORED BAGASSE
}

\author{
C. MACASKILL ${ }^{1}$, M. J. SEXTON ${ }^{1}$ and B. F. GRAY ${ }^{2}$
}

(Received 14 December, 1998; revised 4 April, 1999)

\begin{abstract}
The storage of bagasse, which is principally cellulose, presents many problems for the sugar industry, one of which is bagasse loss due to spontaneous combustion. This is an expensive problem for the industry as bagasse is used as a fuel by sugar mills, and for cogeneration of electricity. Self-heating occurs in the pile through an oxidation mechanism as well as a moisture dependent reaction. The latter reaction is now known to exhibit a local maximum, similar to the heat release curves found in cool-flame problems. Bagasse typically contains $45-55 \%$ by weight of water when milling is completed and the question of how to reduce the moisture content is important for two reasons. Firstly, wet bagasse does not burn nearly as efficiently as dry bagasse, and secondly, self-heating is greatly enhanced in the presence of water, for temperatures less than $60-70^{\circ} \mathrm{C}$.

An existing mathematical model is used, but modified to take into account the newly observed peak in the moisture dependent reaction. Most of the previously reported complex bifurcation behaviour possible in this model is not realized when physically realistic parameter values are used. The bifurcation diagram describing the long-time steady-state solution is the familiar S-shaped hysteresis curve. In the presence of the new form of the moisture dependent reaction, an intermediate state can be found which is not a true steady-state of the system as, in reality, the characteristics of the pile slowly change as water is lost. This state corresponds to observations of an elevated temperature (around $60-70^{\circ} \mathrm{C}$ ) which persists for long periods of time. Approximate equations can then be defined which predict this intermediate state, and hence a different hysteresis curve is found. A simple explanation for the process by which water is lost from the pile is obtained from these equations and an analytical expression is given for the exponential decay of water levels in the pile.
\end{abstract}

\section{Introduction}

The Queensland sugar industry produces many millions of tonnes of sugar every year. A by-product of this processing is bagasse, the material (largely cellulose) that remains after sugar cane has been milled and the sugar removed. Large quantities of

\footnotetext{
'School of Mathematics and Statistics, The University of Sydney, NSW 2006, Australia.

${ }^{2}$ School of Chemistry, Macquarie University, Sydney, Australia.

(C) Australian Mathematical Society 2001, Serial-fee code 0334-2700/01
} 
bagasse are produced over the period during which the sugar is being extracted from the sugar cane, stored on site for an extended period and eventually used as fuel for the sugar mills, often in the next milling season. Dry bagasse, which burns at a higher temperature, is a superior fuel to wet bagasse. However, because near-boiling water is used to remove the last of the sugar from the cane, when bagasse leaves the sugar mill it has a water content of up to $55 \%$ by weight and a temperature around $50^{\circ} \mathrm{C}$.

Consequently, as wet bagasse does not burn nearly as effectively or efficiently as dry bagasse, burning as it does at a lower temperature, there are direct economic and environmental costs in using the wet material. Stockpiling wet bagasse also risks spontaneous ignition of the pile or the leaching of noxious acids into the surrounding earth. Clearly then, understanding the way in which bagasse heaps dry out is an extremely important issue for the sugar industry. Furthermore, it is desirable to use the self-heating of the bagasse pile to enhance the reduction of moisture content during storage, while at the same time ensuring that spontaneous combustion cannot occur.

Early research work $([1,3])$ concentrated on the combustion of dry bagasse, which is initiated by the oxidation process. Although this gave useful information on the use of bagasse as a fuel, it does not give a complete picture of the behaviour of hot, wet stockpiles of bagasse. Wet cellulose, which is the principal component of bagasse, ignites more easily than dry cellulose. Recent experimental work [6] demonstrates that cellulose undergoes an exothermic reaction with water at moderate temperatures. This reaction is thought to be possibly of a hydrolytic nature, though it is not well understood at present. A simple model of the bulk effect with the reaction rate proportional to the water concentration, with a maximum at approximately $55^{\circ} \mathrm{C}$, consistent with empirical pile measurements, will be used here. (We refer henceforth to the oxidation reaction as the 'dry' reaction, and the moisture dependent reaction as the 'wet' reaction.)

It is inconsistent to study these wet reaction effects without also considering evaporation and condensation of water, and the diffusion of water vapour through the pile, [4]. For example, when a hot wet pile of bagasse is exposed to the cooler temperature external to the pile, heat is conducted out towards the edge of the pile. As this happens, the water, which is initially locally at equilibrium, condenses in response to the lower temperature, giving out heat. At the same time the wet reaction and the dry reaction give rise to more heat output. This is partially balanced by local evaporation, which requires the input of heat. There is then a fine balance between these competing effects.

In this paper, we take the model equations of $[8,9]$ with some minor modifications, principally to model more closely the form of the wet reaction term. We then consider piles varying in one spatial dimension only, which considerably eases the numerical and mathematical difficulties, while still retaining most of the physically relevant processes. The actual physical parameters are assigned values closely corresponding 
to those encountered in practice, so the range of bifurcation behaviour possible is considerably reduced from that discussed in [8] where a general analysis of the bifurcation behaviour is given. Indeed, in the absence of the wet reaction we find only the standard S-shaped hysteresis curve, with a quiescent lower stable state, an intermediate unstable state and an upper stable state corresponding to the burning state. However, when the wet reaction term is included we find new behaviour, which corresponds to observations and may be useful in practice for enhancing the drying out of bagasse piles. Because the measured form of heat release corresponding to the wet reaction is such as to introduce a local maximum in the heat release curve, we find an additional approximately steady-state temperature at around $60-70^{\circ} \mathrm{C}$. This is not a true steady-state solution of the equations as it typically persists for a limited time, of order months to years, and furthermore, the water levels in the pile typically change significantly over this period. The elevated temperature state does not persist for all times as the heat-release curve maximum, present because of the wet reaction, diminishes in time as the percentage of water in the pile reduces. Eventually a critical value of the water mass is reached at which the intermediate temperature state can no longer be supported, and the temperature (effectively) bifurcates, usually to the quiescent steady-state.

We find that with initial conditions that correspond closely to those found in practice, for a limited range of pile thicknesses (typically between 1 and $5 \mathrm{~m}$ ) the intermediate state will be established very quickly, on a fast timescale of the order of days. Over this time the liquid water level in the pile remains close to constant, although there is considerable relative change in the profile and magnitude of the (small) part of the water that is in vapour form. If we make the assumption that the liquid water level is exactly constant, then we can write down approximate steady-state equations for the spatially discretised temperature and water vapour levels, which are then amenable to standard bifurcation analysis. The intermediate state is then a true steady-state of these approximate equations. Furthermore, if we make the assumption that the temperature profile remains unchanged at these values until the time is reached at which the intermediate steady-state can no longer be supported, it is possible to calculate an approximate form for the evolution of the water level in the pile, using simple linearised equations. (Physically, the self-heating of the pile means that liquid water evaporates more quickly than the water vapour condenses, with excess vapour being removed from the pile by diffusion.) These approximate solutions allow us to estimate the time at which the temperature will undergo a steep drop (that is, approximately a bifurcation). This time is very important from a practical point of view since it is possible that after the 'bifurcation', the liquid water levels may increase. The temperature has now adjusted to a near ambient stable state, and at these values it is not sufficient to drive off more water. The moisture content of the pile may increase as the water levels attempt to meet steady-state conditions consistent with 
the water vapour levels in the surrounding atmosphere. As we are concerned with the minimisation of the moisture content, it is clear that the pile should be covered, or utilised, at the point of 'bifurcation'. Indeed, this kind of behaviour has recently been observed in an experimental pile [6].

The plan of the paper is as follows. First we give the PDEs describing the evolution of temperature (that is, the energy equation) and the liquid and vapour water levels. In particular we describe the modifications to the model of [8] required to model properly the wet reaction. A short description of the numerical technique used to integrate the full set of equations will be given, followed by representative results for a range of pile widths, illustrating the parameter range in which the intermediate temperature state is achieved. Next, we describe the bifurcation analysis of the approximate equations, valid when the liquid water levels are constant, and then describe how the governing equations for the water levels can be decoupled and approximately linearised using the results of this bifurcation analysis. These approximate results will then be compared with the full solutions. Finally, we present conclusions and explain how this model must be modified to deal with full-scale bagasse piles, where it is thought that the effects of oxygen depletion must be included.

\section{Mathematical formulation}

Consider a (one-dimensional) bagasse pile of thickness $w$, where the spatial coordinate $\boldsymbol{x}^{\prime} \in[-w / 2, w / 2]$. This is the simplest case that demonstrates the relevant physical phenomena, and is of particular interest as Halliburton [6] has recently built a vertical bagasse pile, of height and width of order $10 \mathrm{~m}$ and of thickness $1.2 \mathrm{~m}$, for which a one-dimensional model is appropriate. Detailed comparisons with his measurements will be made in a future paper.

As our starting point, we take the three variable system of PDEs formulated in [8] where the dimensional variables are water, in liquid and vapour form, and temperature, denoted by $X^{\prime}, Y^{\prime}$ and $T$ respectively. In order to model the bagasse problem more accurately, we make several modifications. The first generalisation is to model the density of the pile of bagasse as a function of the liquid water level. In this manner, the quantity $\rho c\left(=C_{s}\right.$ in the notation of [8]) becomes $\rho c=\rho_{b} c_{b}+m_{w} X^{\prime} c_{w}$, where $c_{b}$ and $c_{w}$ are the heat capacities of dry bagasse and water respectively, $m_{w}$ is the molecular weight of water, $\rho_{b}$ is the density of dry bagasse, $\rho$ is the total density and $c$ is the total heat capacity.

To deal with a variety of boundary conditions corresponding to those found in practice, it is found that scaling the liquid and vapour water levels with respect to their initial levels, as was done in [8] is not very convenient. Accordingly, the water levels have been scaled using $X=X^{\prime} m_{w} / \rho_{b}$ and $Y=Y^{\prime} m_{w} / \rho_{b}$. 
The major modification is that the wet reaction term, modelled in [8] with an Arrhenius form, has been changed to take account of preliminary experimental results obtained in [6]. There it was found that there is a peak in the wet reaction at around $55^{\circ} \mathrm{C}$, followed by a very sharp drop at higher temperatures. The model used here is accordingly:

$$
\text { rate of wet reaction }=X^{\prime} \exp \left(-E_{w} / T\right) f(T)
$$

with $f(T)=\left\{\tanh \left[r_{1}\left(r_{2}-T_{1}\right)\right]+1\right\} / 2$. The two constants in the function $f(u)$ take the values $r_{1}=0.6$ and $r_{2}=58$, and $T_{1}=T-273$. The constants $E$ and $E_{w}$ represent the activation energies of the dry and wet reactions respectively, and $R$ is the universal gas constant. The wet reaction rate is assumed to be directly proportional to the scaled liquid water concentration as in [8].

The corresponding scaled equations describing the time evolution of the liquid and vapour concentrations $X$ and $Y$, and of the temperature $u=R T / E$, are

$$
\begin{aligned}
\left(\beta_{1}+\beta_{2} X\right) \frac{\partial u}{\partial t}= & \beta_{3} \exp (-1 / u)+\beta_{4} X \exp \left(-\alpha_{w} / u\right) f(u) \\
& +\alpha\left[\phi_{c} Y-\phi_{e} X \exp (-\alpha / u)\right]+\eta \nabla^{2} u, \\
\frac{\partial Y}{\partial t}= & \phi_{e} X \exp (-\alpha / u)-\phi_{c} Y+\gamma \nabla^{2} Y, \\
\frac{\partial X}{\partial t}= & -\phi_{e} X \exp (-\alpha / u)+\phi_{c} Y,
\end{aligned}
$$

where the time has been scaled by $t=t^{\prime} Z$ and the spatial coordinate $x$ scaled by the pile thickness $w$, that is, $x=x^{\prime} / w$.

The oxidation and moisture dependent reactions are the first and second terms respectively on the right-hand side of the energy equation. The effects of condensation and evaporation on the temperature have been accounted for in the energy equation by the third and fourth terms respectively, and these terms also appear in the mass balance equations for liquid and vapour water concentration levels. Diffusion is considered for both heat and water vapour, whereas the diffusion of water in the liquid state is assumed not to be significant.

The non-dimensional constants in the governing equations (2) are

$$
\begin{aligned}
& \alpha=L_{v} / E, \quad \alpha_{w}=E_{w} / E, \quad \phi_{c}=Z_{c} / Z, \quad \phi_{e}=Z_{e} / Z, \\
& u_{a}=R T_{a} / E, \quad Y_{a}=m_{w} Y_{a}^{\prime} / \rho_{b}, \quad \beta_{1}=c_{b} m_{w} / R, \quad \beta_{2}=c_{w} m_{w} / R, \\
& \beta_{3}=Q m_{w} / E, \quad \beta_{4}=Q_{w} \rho_{b} Z_{w} / Z E, \quad \eta=k m_{w} / \rho_{b} Z R w^{2}, \quad \gamma=D / Z w^{2} .
\end{aligned}
$$

The first six constants are identical to the formulation in [8] while the remaining constants account for the fact that the pile density is now a function of the liquid water 
level and also gives the new scaling on the liquid and vapour water concentrations, as noted earlier. The dimensional constants $Q$ and $Q_{w}$ are the exothermicities of the dry and wet reactions respectively, and $Z$ and $Z_{w}$ are the corresponding pre-exponential factors. The thermal conductivity of the material is denoted by $k$ and $L_{v}$ is the latent heat of vaporisation. The values of the dimensional constants are found in the nomenclature section.

To complete the formulation of the bagasse model, the boundary conditions for temperature and water vapour are required. The condition for the scaled temperature $\boldsymbol{u}$ becomes (assuming left-right symmetry)

$$
\frac{\partial u}{\partial x} \pm B i\left(u-\dot{u}_{a}\right)=0, \quad x= \pm 1 / 2,
$$

where $u_{a}$ is the ambient temperature external to the pile and the Biot number is defined as $B i=h w / k$, with $h$ the heat transfer coefficient from the material to the external atmosphere.

Similarly for the water vapour level

$$
\frac{\partial Y}{\partial x} \pm A\left(Y-Y_{a}\right)=0, \quad x= \pm 1 / 2,
$$

where $Y_{a}$ is the ambient water vapour level, and here we assume that $A=B i$. The ambient water vapour level satisfies the condition

$$
Y_{a}=Y_{s}\left(u_{a}\right) \times \text { relative humidity, }
$$

where the relative humidity is expressed as a percentage. The value of $Y_{s}\left(u_{a}\right)$ corresponds to the situation when water is conserved and in steady-state with the ambient conditions. Using the notation of [8], $\lambda$ denotes the percentage of water by weight in the bagasse pile and

$$
Y_{s}\left(u_{a}\right)=\left(1-X_{s}\left(u_{a}\right)\right)=\frac{\phi_{e}}{\phi_{c}+\phi_{e} \exp \left(-\alpha / u_{a}\right)} .
$$

This formulation is different to that in [8] where the boundary condition on the water vapour $Y$ was determined by water conservation, that is, $X+Y=1$, as the pile was closed to the environment. In the absence of any diffusion of $X$, no boundary conditions are required for the liquid water levels.

\section{Numerical treatment of the full system of PDEs}

In order to provide a reliable benchmark for the qualitative and approximate solutions that we discuss later in the paper, an accurate and efficient method of solution for 
the system of equations given above is required. We solve the system of three PDEs (2) with the associated boundary conditions (3) and (4) using the method of lines. A second-order spatial discretisation is used for the diffusive terms and the boundary conditions. The interior spatial nodal points are given by $x_{j}=j \Delta x, j=2, \ldots, N-1$ where $\Delta x=1 /(N-1)$. The $3(N-2)$ simultaneous ordinary differential equations for $u_{j}, Y_{j}, X_{j}, j=2, \ldots, N-1$, are

$$
\begin{aligned}
\left(\beta_{1}+\beta_{2} X_{j}\right) \frac{d u_{j}}{d t}= & \beta_{3} \exp \left(-1 / u_{j}\right)+\beta_{4} X_{j} \exp \left(-\alpha_{w} / u_{j}\right) f\left(u_{j}\right) \\
& +\alpha\left[\phi_{c} Y_{j}-\phi_{e} X_{j} \exp \left(-\alpha / u_{j}\right)\right]+\eta \frac{u_{j-1}-2 u_{j}+u_{j+1}}{\Delta x^{2}}, \\
\frac{d Y_{j}}{d t}= & \phi_{e} X_{j} \exp \left(-\alpha / u_{j}\right)+\phi_{c} Y_{j}+\gamma \frac{Y_{j-1}-2 Y_{j}+Y_{j+1}}{\Delta x^{2}}, \\
\frac{d X_{j}}{d t}= & -\phi_{e} X_{j} \exp \left(-\alpha / u_{j}\right)+\phi_{c} Y_{j}
\end{aligned}
$$

These equations must be integrated to find the time and spatial evolution of the dependent variables. The differential equations, which turned out to be stiff in most cases, were solved with the MATLAB variable time step ODE solver ode23 s.m.

\section{A typical case}

The behaviour of a bagasse pile for a given set of initial conditions is dependent on its thickness. A pile may ignite if the rate of heat loss through the boundary to the environment is less than the rate of heat production within the pile. The heat lost through the boundary is dependent on the heat and water vapour diffusion coefficients, $\eta$ and $\gamma$ respectively, which are both functions of the width $w$. (See the non-dimensionalisation scheme earlier.) In the well-stirred approximation as used in [8], the heat loss coefficient $L=\eta B i$, is inversely proportional to the width $w$. Therefore, increasing the width will result in a decrease in the rate of heat removal. The heat diffusion coefficient $\eta$ also depends on the density of dry bagasse, $\rho_{b}$. Its precise value is not known, but can vary between the limits $100<\rho_{b}<190 \mathrm{~kg} \mathrm{~m}^{-3}$, depending on the packing. Here, we take $\rho_{b}=125 \mathrm{~kg} \mathrm{~m}^{-3}$.

The initial conditions for the problem used here are similar to those encountered in a bagasse pile soon after the processing is completed in the mill. That is, the initial bagasse temperature is $55^{\circ} \mathrm{C}(u(0)=0.02523)$ and initial water content is $50 \%$ $(X(0)=1)$, with an ambient external temperature of $30^{\circ} \mathrm{C}\left(u_{a}=0.0233\right)$. The initial water vapour level $Y(0)$ is set by requiring initial equilibrium with the corresponding given value of $X(0)$, that is, $Y(0)=\phi_{e} \exp (-\alpha / u(0)) / \phi_{c}$. The ambient external water vapour level $Y_{a}$ corresponds to a relative humidity of $70 \%$, typical of conditions in 
Northern Queensland, and in addition, it is assumed that the initial temperature and water concentrations are spatially uniform across the pile, as would occur in practice.

Three possible states of this model are shown in Figure 1 for varying values of the pile thickness $w$, with initial conditions as described above. Figure 1 (a) gives the time evolution of the centre pile temperature (in ${ }^{\circ} \mathrm{C}$ ) when the pile thickness is $1.5 \mathrm{~m}$. A small pile width allows the heat to escape quickly through the boundaries, and this is indicated on Figure 1 (a) by the fact that the temperature drops from its initial value of $55^{\circ} \mathrm{C}$ to a near ambient temperature within about 50 days. Figure 1 (b) shows the corresponding water levels slowly approaching their steady-state values. The steady-state value for the water vapour concentration is found to be $Y_{s}=Y_{a}$ uniformly across the pile, with the liquid water steady-state concentration being $X_{s}=$ $\phi_{c} Y_{a} \exp \left(\alpha / u_{s}\right) / \phi_{e}$, where $u_{s}$ is the (spatially dependent) steady-state temperature at the centre of the pile, slightly above $u_{a}$. This case shown in Figures 1 (a) and (b) would not be desirable in practice as the water levels are still high after one year and the bagasse in this form is not desirable as a fuel. However, the pile shows no tendency to ignite.

The case where the burning combustion state is achieved is shown in Figures 1 (e) and (f) for the pile thickness of $5 \mathrm{~m}$. The temperature and scaled water vapour level $Y / Y_{\max }$ (shown with a dashed line) follow a continual upward trend over the given time interval. After a period of 30 days, the temperature reaches $100^{\circ} \mathrm{C}$ (the boiling point of water) whereupon the model fails. (Evaporation and condensation of water are only modelled properly here for temperatures below $100^{\circ} \mathrm{C}$.) At this point, the temperature within the pile would continually increase eventually leading to the burning state. This is clearly an undesirable scenario.

Of particular interest, practically, is the case shown in Figures 1 (c) and (d). Observations have shown that real bagasse piles tend to remain at an approximately constant, elevated temperature for considerable periods of time. Bagasse pile temperatures have been gathered from a number of mills in Northern Queensland and summarised in [1]. Elevated temperatures of approximately $64^{\circ} \mathrm{C}$ have been noted lasting up to 180 days. More recently, in a carefully monitored experiment using a pile of small thickness $(1.2 \mathrm{~m})$, Halliburton [6] found the centre temperature remained at about $60^{\circ} \mathrm{C}$ for nearly six months. There are practical advantages in maintaining the pile at such temperatures as the rate of drying out is considerably enhanced. Therefore it is of interest to consider cases where this state is achieved. It was found that the elevated temperature state (we refer to this as a quasi-steady-state) could be achieved with a pile thickness of $2.2 \mathrm{~m}$, with initial conditions described above. The resultant curves for (unscaled) temperature, and for the water levels $X$ (liquid) and $Y / Y_{\max }$ (vapour) (shown with a dashed line) are given as a function of time in Figures 1 (c) and (d).

There are several important features apparent in this particular case. First consider Figure 2 which shows the behaviour in Figures 1 (c) and (d) over a shorter time interval 


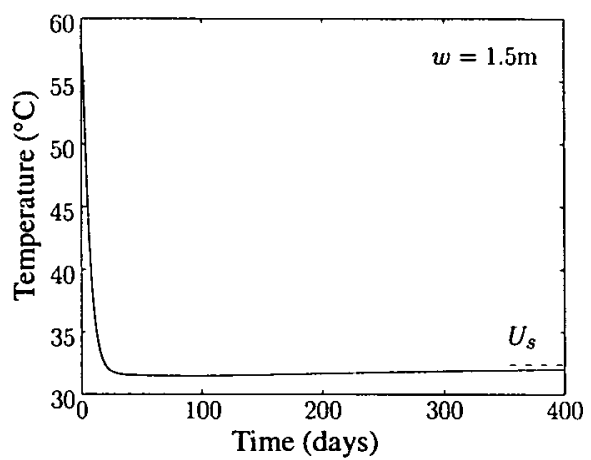

(a)

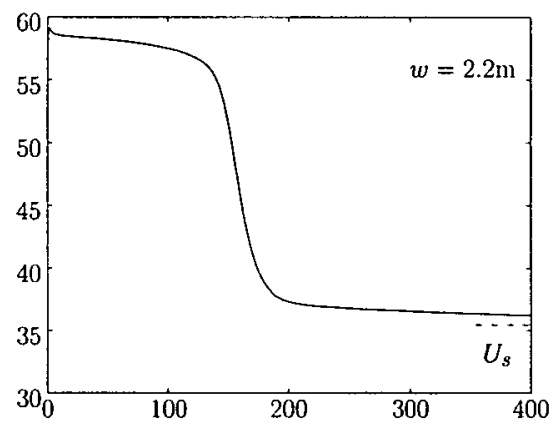

(c)

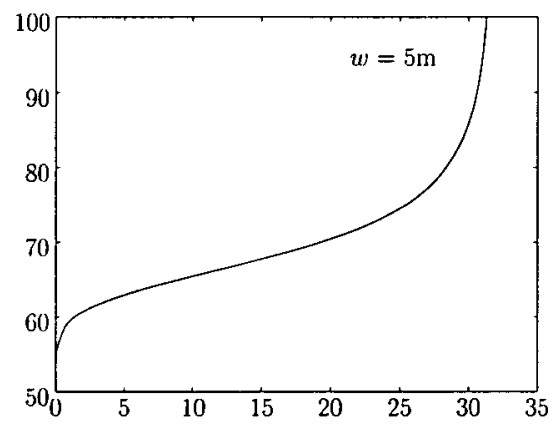

(e)

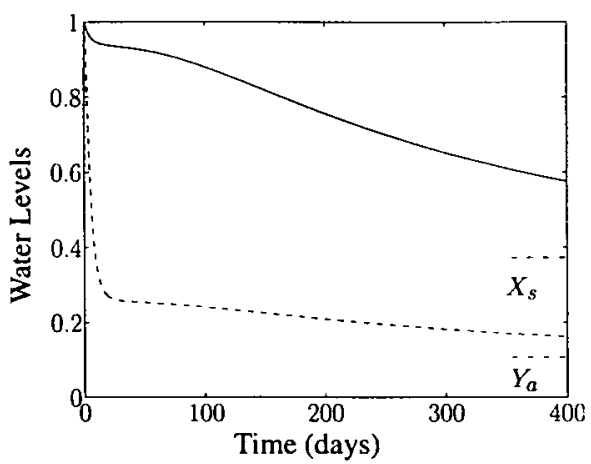

(b)

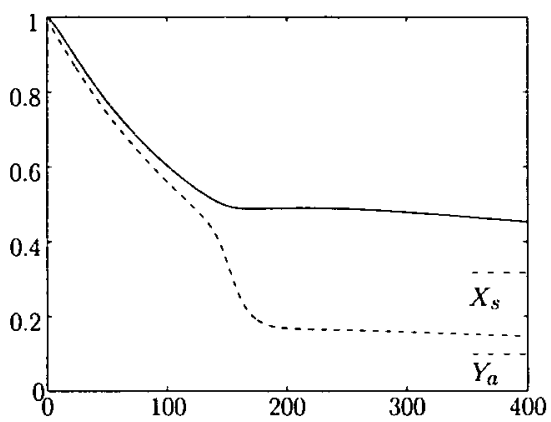

(d)

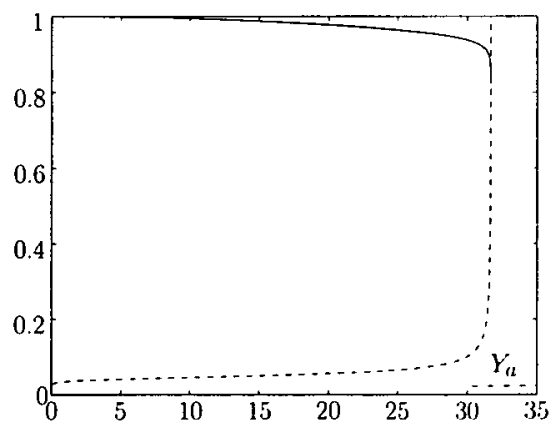

(f)

FIGURE 1. The time variation of temperature (a), (c), (e) and liquid water levels $X(-)$ and scaled vapour $Y / Y_{\max }(--)(\mathrm{b}),(\mathrm{d})(\mathrm{f})$, at the centre of a bagasse pile of thickness $w=1.5,2.2$ and $5 \mathrm{~m}$ respectively. Steady-state values are indicated on each figure by a dot-dash line. 


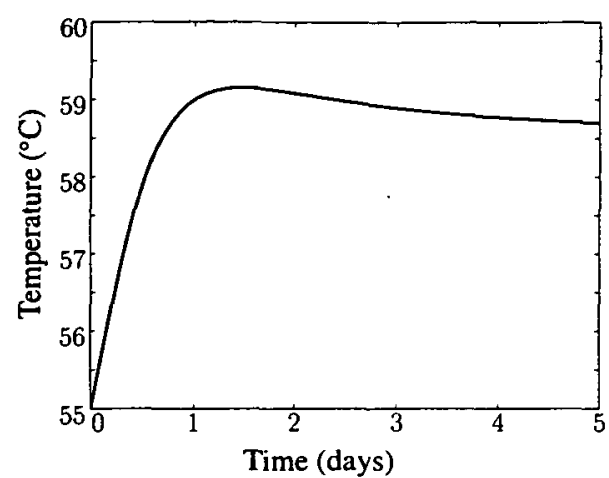

(a)

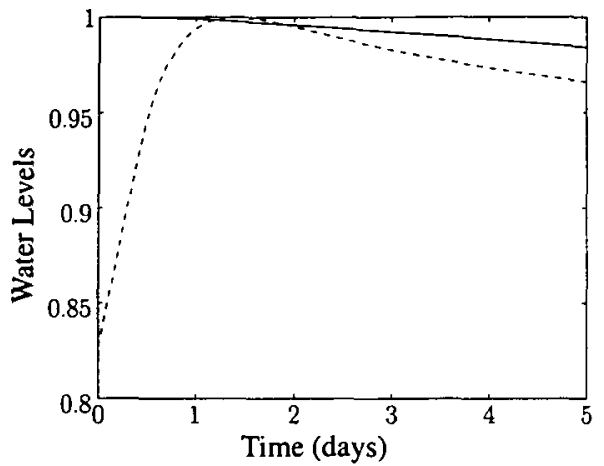

(b)

FIGURE 2. The time variation of (a) temperature and (b) liquid water $X(-)$ and scaled water vapour $Y / Y_{\max }(--)$ levels at the centre of a bagasse pile of thickness $w=2.2 \mathrm{~m}$.

of five days to illustrate the initial behaviour. In Figure 2 (a) the temperature adjusts very quickly (over several days) to the quasi-steady-state of approximately $59^{\circ} \mathrm{C}$. Interestingly, this short time scale is noted in [1] where it has been found that an initial rapid heating rate occurs over two days. On the same initial fast time-scale as the temperature adjustment, the water vapour level $Y / Y_{\max }$ rises quickly to its maximum, indicated in Figure 2 (b), while the liquid water level $X$ remains constant. Over the next 6 months or so, the temperature remains at or near the quasi-steady-state temperature while the water levels, both liquid and vapour, exponentially decay as indicated in Figure 1 (d). When the minimum level of $X$ is reached (at about 200 days), the wet reaction (for which the heat release is proportional to $X$ ) has decreased to the extent that the quasi-steady-state temperature can no longer be sustained and the temperature drops very quickly to a level close to its true long-time steady-state value. This is essentially a bifurcation phenomenon, in a sense that we will quantify later. At this point, the $X$ and $Y$ levels approach their true steady-states, $X_{s}$ and $Y_{s}$ respectively.

The evolution of the spatial dependence of temperature and water levels $X$ and $Y / Y_{\max }$ is shown in Figure 3, corresponding to the example in Figures 1 (c) and (d). The temperature and vapour assume close to parabolic form in the initial adjustment to the quasi-steady-state ( $t=2$ days), while $X$ remains initially constant across the pile. The parabolic form for the temperature is not surprising, as that is what is found for example for the linear heat conduction problem, where such behaviour can be identified as the leading order term in the long time expansion of the Laplace transform solution of the problem.

4.1. Interpretation and analysis It is possible, under some assumptions, to interpret most of the features of the cases described above. For the present, the effects of evaporation and condensation are ignored, $X$ is assumed constant, as it is for short 


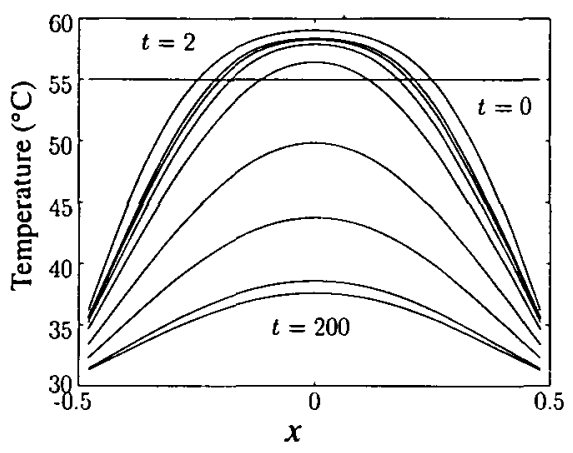

(a)

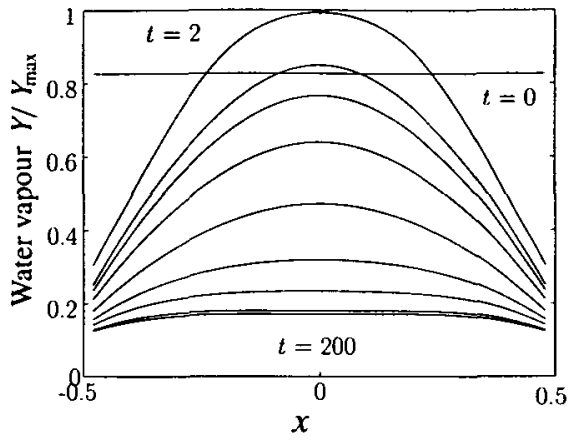

(b)

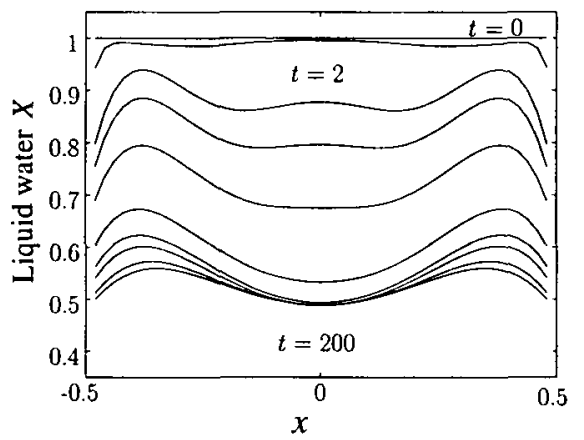

(c)

FIGURE 3. Spatial profiles of (a) temperature, (b) scaled water vapour $Y / Y_{\max }$ and (c) liquid water $X$ for the spatial coordinate $x \in[-0.5,0.5]$.

times (see Figure 2 (b)) and the heat conduction term is approximated with a bulk heat removal term $L\left(u-u_{a}\right)$, where $L$ will be taken to be some measure of the heat loss which will be inversely proportional to the thickness of the pile $w$, as described earlier. The heat release (the dry reaction plus the wet reaction) and the heat removal term $L\left(u-u_{a}\right)$ have been plotted in Figure 4 as a function of $u$.

The unusual feature of the heat release curve is the local maximum, corresponding to the maximum in the wet reaction term, (1). For a fixed $X$ level, there can be as many as five steady-states, three of which are stable. (Four steady-states are evident in Figure 4, with the fifth being the stable burning state found at very high temperatures.) A similar heat release curve is found in the so-called cool flame problem, described by [5]. However, in the cool flame problem, it is found that the intermediate steady-state is not in fact stable for all values of $L$, due to the presence in some cases of Hopf bifurcations. In the well-stirred approximation to this problem, it can be proved that under certain conditions, periodic solutions cannot occur (see appendix for details.) Similarly, in the spatially distributed case, (2), we have found no periodic solutions, 


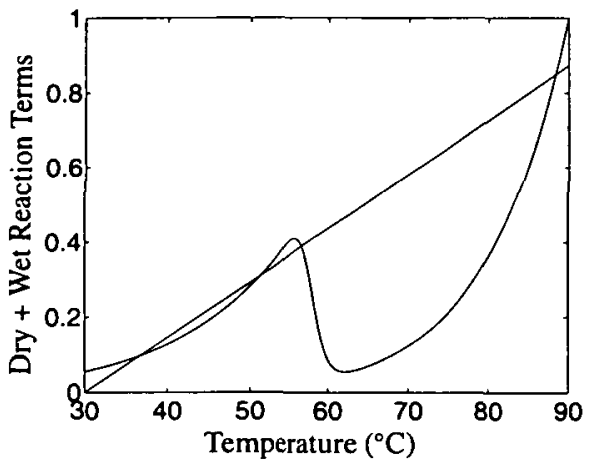

FIGURE 4. The heat release mechanism described by the dry reaction plus wet reaction and the bulk heat loss term shown as a function of temperature scaled by the value at $90^{\circ} \mathrm{C}$.

although it has not been proved that they do not exist.

The low-temperature stable solution corresponds to near-ambient conditions, while the high-temperature stable solution corresponds to the burning state. The intermediate stable state, only present because of the special form of the wet reaction term, corresponds to the quasi-steady-state observed in the full numerical result discussed above, and, we believe, often observed in real bagasse piles. We call this a quasi-steady-state because in reality $X$ evolves slowly in time. The wet reaction term typically decreases in magnitude until the intermediate intersection is no longer present. A similar heat release curve is found in the model considered by [2] where the intermediate steady-state shows variable stability. There the intermediate steady-state is necessary to allow the direct partial oxidation of methane and here this intermediate state enhances the drying out process of the pile.

\subsection{Approximate solution for the water content of the pile as a function of time}

In the previous section the essence of the physical mechanisms taking place were described. However, some further, more quantitative progress can be made using the same basic ideas. We scale $X, Y$ and $u$ with their steady-state values at the centre of the pile, $X_{s}, Y_{s}$ and $u_{s}$ respectively, so that $\hat{X}=X / X_{s}, \hat{Y}=Y / Y_{s}$ and $\hat{u}=u / u_{s}$.

Rewriting (2) gives

$$
\begin{aligned}
\left(\frac{\beta_{1}}{X_{s}}+\beta_{2} \hat{X}\right) \frac{\partial \hat{u}}{\partial \hat{t}}= & \epsilon\left\{\frac{\beta_{3}}{\phi_{e} X_{s}} \frac{\exp \left[-\alpha(1 / \alpha \hat{u}-1) / u_{s}\right]}{u_{s}}\right. \\
& +\frac{\beta_{4}}{\phi_{e}} \frac{\exp \left[-\alpha\left(\alpha_{w} / \alpha \hat{u}-1\right) / u_{s}\right]}{u_{s}} \hat{X} f\left(u_{s} \hat{u}\right) \\
& \left.+\frac{\alpha}{u_{s}}[\hat{Y}-c(\hat{u}) \hat{X}]+\frac{\eta}{\epsilon \phi_{c} X_{s}} \nabla^{2} \hat{u}\right\}
\end{aligned}
$$




$$
\begin{aligned}
& \partial \hat{Y} / \partial \hat{t}=-[\hat{Y}-c(\hat{u}) \hat{X}]+\left(\gamma / \phi_{c}\right) \nabla^{2} \hat{Y} \\
& \partial \hat{X} / \partial \hat{t}=\epsilon[\hat{Y}-c(\hat{u}) \hat{X}]
\end{aligned}
$$

where $c(\hat{u})=\exp (-\alpha(1 / \hat{u}-1)), \hat{t}=\phi_{c} t$ and $\epsilon=\phi_{e} \exp \left(-\alpha / u_{s}\right) / \phi_{c}=Y_{s} / X_{s}$ is a small quantity. All terms on the right-hand side of the equation for $\hat{u}$ are small, including the heat conduction term, where we note that $\eta / \epsilon \phi_{c} \ll 1$. The appearance of two timescales in the example run in Figures 1 (c) and (d) can now be interpreted: the fast timescale is $\hat{t}$ while the slow time is $\tau=\epsilon \hat{t}$. On the fast timescale $\hat{X}$ is constant in time, since the right-hand side of the $\hat{X}$ equation is approximately zero. At longer times of $O(1 / \epsilon)$, terms of $O(\epsilon)$ on the right hand side of the $\hat{X}$-equation can no longer be ignored and thus $\hat{X}$ slowly decays. An approximate analytical picture can now be put together, in which the intermediate quasi-steady-state plays a crucial role. If we look for the steady-states of (5), with $\partial \hat{X} / \partial \hat{t}=0$ then, indeed, our quasisteady-state is a true steady-state of the approximate equations and can be found by standard techniques (for example, Newton-Raphson methods, or with the aid of the AUTO97 package). There is a minor complication in that we are dealing with PDEs, but we just solve for steady states of the corresponding spatially discretised equations for $\hat{u}$ and $\hat{Y}$.

At this zeroth order approximation, $\hat{X}$ plays the role of a bifurcation parameter. If the other parameters in the problem are such that the intermediate stable state can be achieved, further progress can then be made, as on the slow timescale $\hat{u}$ now remains approximately constant in time, with a roughly parabolic profile in space. The approximate constancy of $\hat{u}$ on the slow timescale $\tau$, as seen in Figure 1 (c) is due to the fact that the wet reaction decays very rapidly at around $60^{\circ} \mathrm{C}$ (see Figure 4). Thus when $\hat{X}$ reduces, so reducing the magnitude of the wet reaction term, the corresponding decrease in $\hat{u}$ is very small. We expect this picture to break down once the intermediate stable state no longer exists, that is, once the liquid water level $\hat{X}$ drops sufficiently. It is at this point that the temperature will bifurcate to its lower stable state and then the $\hat{X}$ and $\hat{Y}$ values will cease to decrease (Figure 1 (d)) or may in some cases start to rise again.

The (now linear) equations for the evolution of $\hat{Y}$ and $\hat{X}$ on the slow timescale $\tau$ can now be solved, once $\hat{u}$ is known and assumed constant in time. In order to make further quantitative progress we then further assume that $\hat{X}, \hat{Y}$ and $\hat{u}$ all have parabolic profiles (see Figure 3 ). The centre value $\hat{u}_{c}$ can be calculated from a bifurcation analysis of the approximate equations that follow when $\hat{X}=$ constant in (5). The parabolic form for $\hat{u}$, satisfying the boundary conditions (3), will have the constant form

$$
\hat{u}_{q s}=\hat{u}_{c}+\frac{B i\left(\hat{u}_{a}-\hat{u}_{c}\right)}{(1+B i / 4)} x^{2},
$$

corresponding to the intermediate stable state. 
We must then solve the linearised equations for $\hat{X}$ and $\hat{Y}$, taking $\hat{u}=u_{q s}$, that is,

$$
\begin{aligned}
\epsilon \partial \hat{Y} / \partial \tau & =-\left[\hat{Y}-c\left(\hat{u}_{q s}\right) \hat{X}\right]+\left(\gamma / \phi_{c}\right) \nabla^{2} \hat{Y} \\
\partial \hat{X} / \partial \tau & =\hat{Y}-c\left(\hat{u}_{q s}\right) \hat{X} .
\end{aligned}
$$

Thus it is necessary to determine the centre water vapour level $\hat{Y}_{c}(\tau)$ as a function of the slow time $\tau=\epsilon t$, where

$$
\hat{Y}=\hat{Y}_{c}(\tau)+\hat{Y}_{1} x^{2}
$$

in order that $\hat{Y}$ satisfies the boundary condition (4) and remains parabolic in profile. Similarly

$$
\hat{X}=\hat{X}_{c}(\tau)+\hat{X}_{1}(\tau) x^{2}
$$

where $\hat{X}_{c}$ and $\hat{X}_{1}$ are to be determined. The value of $\hat{Y}_{c}(0)$ is then given by the central value of $\hat{Y}_{q s}$ as determined from the fast time bifurcation analysis, while $\hat{X}(\tau=0)=X(0) / X_{s}$. When (6), (8) and (9) are substituted in the linearised equations (7) and terms of $O(1)$ are equated and higher order terms in $x$ neglected, we find that

$$
\begin{aligned}
\epsilon d \hat{Y}_{c} / d \tau & =-\left[\hat{Y}_{c}-c\left(\hat{u}_{c}\right) \hat{X}_{c}\right]+2\left(\gamma / \phi_{c}\right) \hat{Y}_{1}, \\
d \hat{X}_{c} / d \tau & =\hat{Y}_{c}-c\left(\hat{u}_{c}\right) \hat{X}_{c} .
\end{aligned}
$$

We note that $\hat{Y}_{c}$ depends on $\hat{Y}_{1}$ (from the heat conduction term). However, the equations can be closed by requiring that the boundary conditions for $\hat{Y}$ are satisfied to $O\left(x^{2}\right)$, so that

$$
\hat{Y}=\hat{Y}_{c}(\tau)+\frac{A\left[\hat{Y}_{a}-\hat{Y}_{c}(\tau)\right]}{(1+A / 4)} x^{2},
$$

that is, $\hat{Y}_{1}=A\left[\hat{Y}_{a}-\hat{Y}_{c}(\tau)\right] /(1+A / 4)$. With this approximation, we can then solve (10) exactly. However, the two eigensolutions correspond directly to fast and slow modes, with the fast mode only relevant for short times. Here we choose to suppress the fast mode by setting to zero the term that is of $O(\epsilon)$, that is, the term $\epsilon d \hat{Y}_{c} / d \tau$. With this approximation, and after rewriting $\hat{Y}_{1}$ in terms of $\hat{Y}_{c}$, we can then use the first of the simultaneous equations (10) to express $\hat{Y}_{c}$ in terms of $\hat{X}_{c}$ and hence solve the ODE for $\hat{X}_{c}$. Using the fact that $\phi_{c} \gg 2 \gamma \delta$, and then reverting to the unscaled variables, we find that

$$
X_{c}=\left(X(0)-\frac{\phi_{c} e^{\alpha / u_{c}}}{\phi_{e}} Y_{a}\right) \exp \left[-\left(\frac{2 \gamma \delta \phi_{e} e^{-\alpha / u_{c}}}{\phi_{c}}\right) t\right]+\frac{\phi_{c} e^{\alpha / u_{c}}}{\phi_{e}} Y_{a}
$$




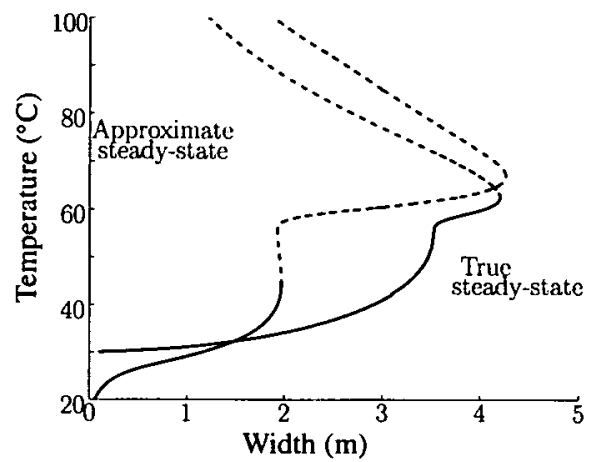

(a)

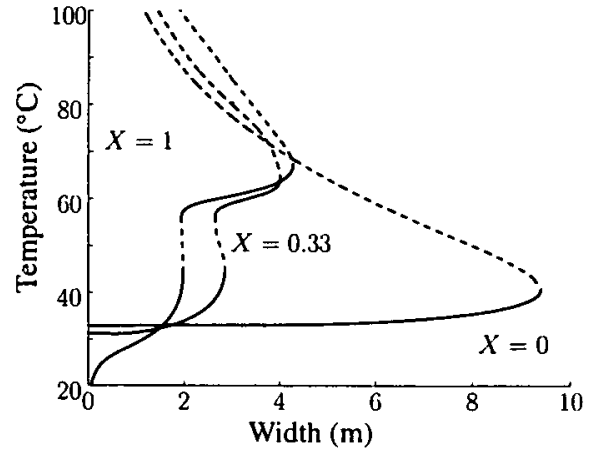

(b)

Figure 5. Bifurcation curves with the width $w$ as the bifurcation parameter: (a) the curve for the approximate equations with $X=1$ and for the full system, where $X=X_{s}$ and (b) curves for the approximate equations with $X=1,1 / 3$, and 0 .

and

$$
Y_{c}=\left(\phi_{e} / \phi_{c}\right) e^{-\alpha / u_{c}} X_{c}
$$

where $\delta=A /(1+A / 4)$.

It is possible to take this approach slightly further. By equating terms out to $O\left(x^{2}\right)$ we can write down differential equations for $\hat{X}_{1}$ and $\hat{Y}_{1}$. The $\hat{Y}_{1}$ equation is inconsistent with our parabolic profile approximations, as the heat conduction term depends on terms of $O\left(x^{4}\right)$ in the expansion for $\hat{Y}$. The equation for $\hat{X}_{1}$ by contrast, can be solved directly, as there is no diffusive term in $\hat{X}$, but we do not provide the details here.

\section{Results}

First we consider the bifurcation behaviour of the equations describing the evolution of temperature and water levels in the bagasse pile, with particular emphasis on the quasi-steady-state, which is practically desirable as it enhances the drying out of the pile.

The equations (5) reduce to a system of two equations when the liquid water level $X$ is considered constant. This allows a direct study of the intermediate quasi-steadystate, which is not in general found as a solution of the full steady-state equations. The steady-state solutions of these approximate equations are shown in Figure 5 (a) where the centre temperature of the pile is plotted as a function of the pile width $w$, here playing the role of a bifurcation parameter. For this case the moisture level is held constant at $50 \%$, that is, $\lambda=0.5$ so that $X=1$. Stable solutions are indicated with solid lines, and unstable solutions with dashed lines. 


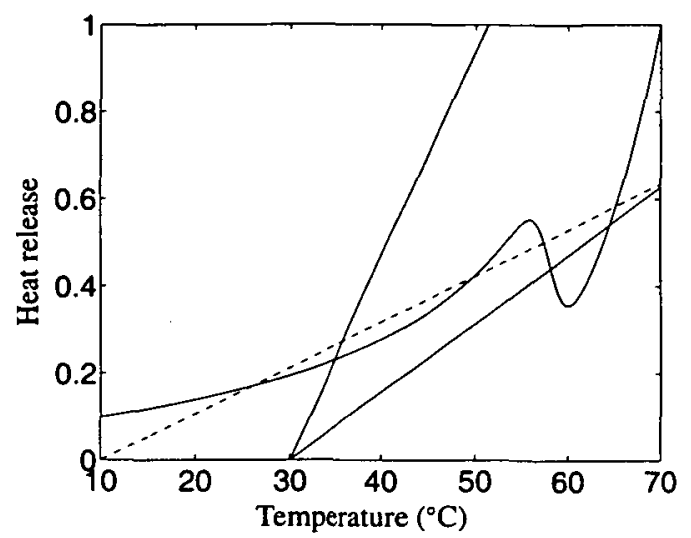

FIGURE 6. The heat release curve, scaled by the value at $70^{\circ} \mathrm{C}$ and using the steady-state liquid water level $X_{s}$, plotted against temperature.

The bottom stable branch, for widths less than $2 \mathrm{~m}$, corresponds to solutions near ambient conditions. The intermediate stable branch, for widths varying between the approximate limits $2<w<4.5 \mathrm{~m}$, corresponds to the quasi-steady-state. (Note that if the density of dry bagasse, $\rho_{b}$, is increased from the value used here, we find that the intermediate stable state can be supported with smaller pile thicknesses.) In order to reduce the moisture content of the pile, it is then desirable to choose the width such that the quasi-steady-state temperature is found. For widths greater than $2 \mathrm{~m}$, the lower stable state cannot be a solution, and for widths greater than $4.5 \mathrm{~m}$, the burning state is found. (This state is not shown on Figure 5 (a), as it occurs for temperatures greater than $100^{\circ} \mathrm{C}$.) There is a narrow region of multistability when the width is given by $1.8<w<1.97 \mathrm{~m}$. In this region, either of the two stable branches can be found with the appropriate initial conditions.

Turning now to Figure 5 (b), the bifurcation diagram for varying values of the initial moisture content is shown for the parameter $w$. As the moisture level decreases, the range of widths for which the quasi-steady-state is achievable changes. With no moisture in the pile $(X=0)$, the intermediate state cannot be realized as the wet reaction term approaches zero in this limit, so that the heat release curve grows monotonically with temperature, and there is no local maximum. This state can almost never be achieved physically as the water level is dependent on the relative humidity level external to the pile. For relative humidity of $70 \%$, as considered here, the minimum moisture level possible is about $25 \%$, that is, $X=1 / 3$.

Returning to Figure 5 (a), the steady-state solution of the full equations (5) (where $X=X_{s}$ ) does not show an intermediate steady state. However, it is clear that the two stable branches have effectively coalesced so that for pile widths between about $3 \mathrm{~m}$ and $4 \mathrm{~m}$ the final state gives a significantly elevated temperature. In order to understand 


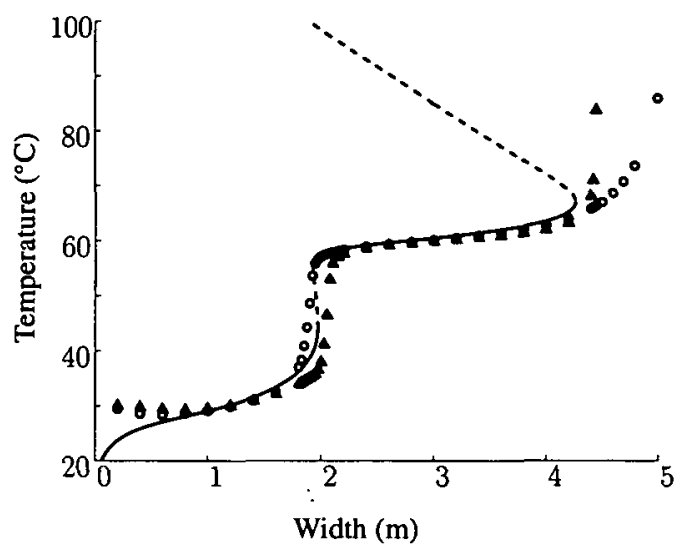

FIGURE 7. Bifurcation curve of the pile centre temperature as a function of the width $w$ from the approximate equations is compared with the numerical results from the full system at time 30 days (o) and at 90 days $(\Delta)$.

this behaviour, we consider Figure 6, which shows the heat release curve at very long time plotted as a function of temperature. Here, $Y=Y_{a}$ and $X=\phi_{c} Y_{a} \exp (\alpha / u) / \phi_{e}$. In this manner, evaporation and condensation balance, that is, $\phi_{c} Y=\phi_{e} X \exp (-\alpha / u)$, and hence the heat release is just the dry plus wet reaction. The solid straight lines represent the heat loss, $L\left(u-u_{a}\right)$, for different values of $L$. This shows that only one steady-state is possible for temperatures less than about $60^{\circ} \mathrm{C}$. In this case, the ambient temperature is $30^{\circ} \mathrm{C}$. However, if the ambient temperature is significantly reduced below this level then the full equations do in fact admit the full range of steady-states shown by the approximate equations. This is indicated by the dashed line in the figure, corresponding to a (low) ambient temperature of $10^{\circ} \mathrm{C}$, where there are once again three intersections, corresponding to stable, unstable and stable states respectively, as was the case in Figure 4.

In Figure 7 we consider the extent to which the approximate bifurcation analysis predicts the actual behaviour as obtained from a full numerical solution of the governing PDEs. In all cases the initial pile temperature was taken to be $55^{\circ} \mathrm{C}$. After thirty days the pile temperature agrees closely with the predictions of the approximate bifurcation analysis. The deviations from the lower steady-state near $w=2 \mathrm{~m}$ are due to the fact that we have not chosen initial conditions that are compatible with this state. Even after ninety days the bifurcation results show good agreement with the integration of the full system of equations, except that at pile widths close to but above $2 \mathrm{~m}$ a near-bifurcation from the intermediate state down to the lower stable state has taken place.

We now show how the approximate solution for the water level at the centre of the pile, derived in the previous section, compares with the full numerical solution (see 


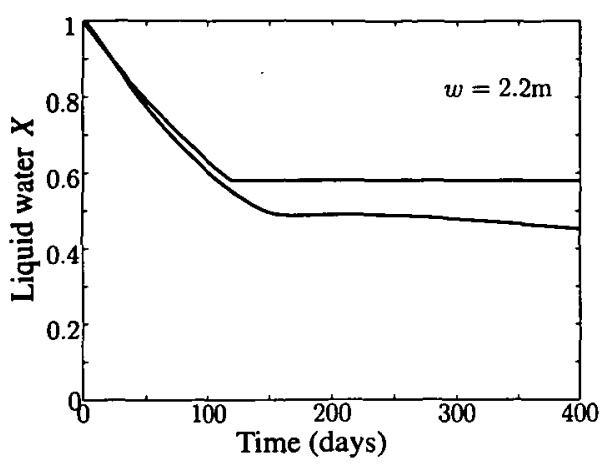

(a)

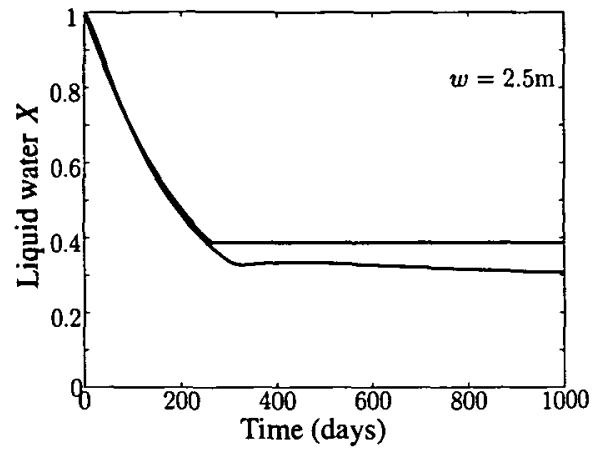

(b)

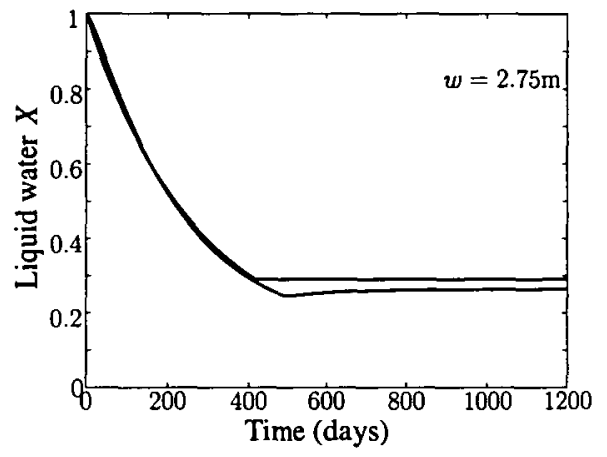

(c)

FIGURE 8. Comparison between numerical and approximate analytic results for (a) $w=2.2 \mathrm{~m}$, (b) $w=$ $2.5 \mathrm{~m}$ and (c) $w=2.75 \mathrm{~m}$.

Figure 8). The exponential form for the analytic solution is used until the time at which $X$ reaches its bifurcation value, as determined from the approximate equations, at which the intermediate steady-state can no longer be supported. We see that the analytic approximation does indeed reflect the actual behaviour quite adequately, although the bifurcation time is underpredicted somewhat, particularly when the width $w$ is small.

In Figure 9, the approximate analytic results for water level at the centre of the pile are given for a range of pile widths, demonstrating the principle that narrow piles dry out more quickly, but that the final water level at the centre of the pile will eventually drop to a lower level for broader piles. It seems reasonable that narrow piles should dry out more quickly. The decreased central value for large piles follows from the fact that the liquid water level is approximately parabolic at large time, with the minimum at the centre, (see Figure 3 (c)), and we expect a roughly similar form for the $X$-profiles near the boundaries for narrow and wide piles at their final steady-states. With these constraints the observed behaviour follows. 


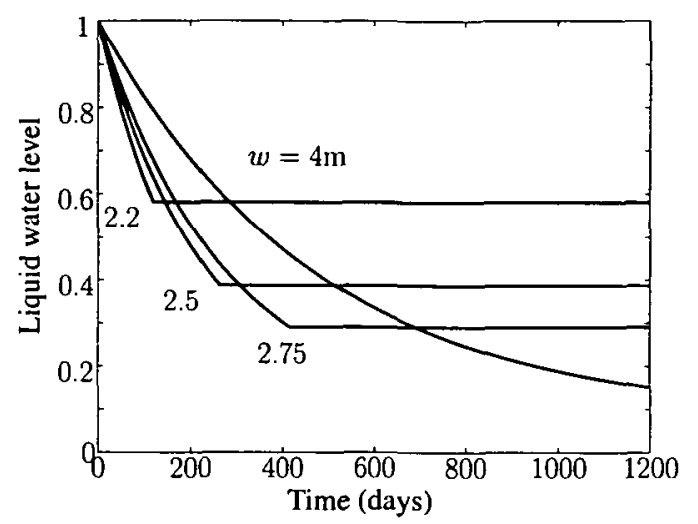

FIGURE 9. Approximate analytic results for $w=2.2,2.5,2.75$ and $4 \mathrm{~m}$.

\section{Conclusions}

It has been known for some time that wet bagasse undergoes a wet reaction which significantly affects the heat release rate. New experimental work [6] has shown that this reaction has a well-defined peak just below $60^{\circ} \mathrm{C}$. In this paper, a straightforward modification to an earlier model [8] has been made, so as to include these new observations. It has been shown that for a one-dimensional bagasse pile, there is a range of widths for which the pile quickly moves to an elevated temperature, near $60^{\circ} \mathrm{C}$, which can be identified as an approximate steady-state of the system. Drying out of the pile is enhanced if this state is maintained over an extended period. In many cases, it turns out that the temperature of the pile changes only slowly in time once the intermediate state is reached. This makes it possible to find a simple analytical expression for the rate at which the bagasse pile dries out, which agrees well with the results from a full numerical integration of the governing equations. Furthermore, an approximate bifurcation analysis has been used to estimate the time at which this drying process ceases, and to determine the range of widths for which the intermediate quasi-steady-state can be attained. This state is desirable as it allows the pile to dry out quickly without undergoing spontaneous combustion. Understanding this process is of commercial importance as dry bagasse is a far better fuel than wet bagasse. One apparently surprising result is that if the width is sufficiently large, the pile will always ignite. In general, this does not comply with observations. It is thought that the oxygen depletion in the interior of the pile must also be considered. This will be the subject of future work.

\section{Acknowledgements}

The authors acknowledge support provided by the SPIRT scheme and Mr Brendan Halliburton for making available data before publication. 


\section{Appendix. Dulac Result}

Consider the well-stirred case approximation to (2) in the regime when $X$ is constant and the diffusion terms are approximated by a bulk heat loss term, as noted earlier:

$$
\begin{aligned}
\frac{\mathrm{d} u}{\mathrm{~d} t} & =\left[\beta_{3} e^{-1 / u}+\beta_{4} X e^{-\alpha_{w} / u} f(u) r+\alpha\left[\phi_{c} Y-\phi_{e} X e^{-\alpha / u}\right]-L_{1}\left(u-u_{a}\right)\right] \\
& =\frac{H(u, Y)}{\beta_{1}+\beta_{2} X}=F(u, Y), \\
\frac{\mathrm{d} Y}{\mathrm{~d} t} & =\phi_{e} X e^{-\alpha / u}-\phi_{c} Y-L_{2}\left(Y-Y_{a}\right)=G(u, Y) .
\end{aligned}
$$

In order to prove the non-existence of oscillations, the Dulac theorem is employed (see [7]). It is necessary to choose a Dulac function $B(u, Y)$ which is positive, continuous and differentiable for all $u$ and $Y$. The Dulac theorem says that if the function $S$ defined by

$$
S=B\left(\frac{\partial F}{\partial u}+\frac{\partial G}{\partial Y}\right)+F \frac{\partial B}{\partial u}+G \frac{\partial B}{\partial Y}
$$

is always of one sign, that is, $S$ never crosses zero, then there are no oscillations.

Let the Dulac function $B=1$, so the function $S$ becomes

$$
\begin{aligned}
S= & \frac{\beta_{3}}{\beta_{1}+\beta_{2} X} \frac{e^{-1 / u}}{u^{2}}+\frac{\beta_{4} X e^{-\alpha_{w} / u}}{\beta_{1}+\beta_{2} X}\left(\frac{\alpha_{w} f(u)}{u^{2}}+f^{\prime}(u)\right) \\
& -\frac{\alpha^{2} \phi_{e}}{u^{2}} \frac{X e^{-\alpha / u}}{\beta_{1}+\beta_{2} X}-\frac{L_{1}}{\beta_{1}+\beta_{2} X}-L_{2}-\phi_{c} .
\end{aligned}
$$

In order to ensure $S$ is negative, it is enough to require that both

$$
\beta_{3} e^{-1 / u} / u^{2}-\phi_{c}\left(\beta_{1}+\beta_{2} X\right)<0
$$

and

$$
\frac{X}{\left(\beta_{1}+\beta_{2} X\right) u^{2}}\left(\beta_{4} e^{-\alpha_{w} / u}\left[\alpha_{w} f(u)+f^{\prime}(u) u^{2}\right]-\alpha^{2} \phi_{e} e^{-\alpha / u}\right)<0
$$

as the loss coefficients $L_{1}$ and $L_{2}$ are positive. The first inequality (A.1) must be satisfied at $u=u_{\max }$ and $X=0$, hence

$$
\beta_{3}<\phi_{c} \beta_{1} u_{\max }^{2} e^{1 / u_{\max }} .
$$

Turning to the second inequality (A.2), let $g(u)=\alpha_{w} f(u)+f^{\prime}(u) u^{2}$, then the maximum value of $g(u)$ is equal to $\alpha_{w}$ as $f^{\prime}(u)<0$ for all values of $u$ and the maximum value $f(u)$ can take is 1 . Rewriting inequality (A.2) as

$$
\beta_{4} \alpha_{w}<\alpha^{2} \phi_{e} \exp \left[\left(\alpha_{w}-\alpha\right) / u\right]
$$


and noting that $\exp \left[\left(\alpha_{w}-\alpha\right) / u\right]>1$, then

$$
\beta_{4}<\alpha^{2} \phi_{e} / \alpha_{w}
$$

Therefore, there are no oscillations in the well-stirred approximation to the bagasse problem when (A.3) and (A.4) are satisfied.

Note this result makes no use of the heat loss coefficients $L_{1}$ and $L_{2}$. For the values of the parameters here, the first inequality becomes $\beta_{3}<7.3 \times 10^{4}$, using the physical maximum on temperature, $u_{\max }=100^{\circ} \mathrm{C}$, and the second inequality is $\beta_{4}<3.9 \times 10^{-5}$. As it turns out, $\beta_{3}=2.8333$ and $\beta_{4}=7.083 \times 10^{-6}$ and therefore, the two constraints (A.3) and (A.4) are satisfied, indicating that the well-stirred system cannot show oscillations.

\section{Nomenclature}

$$
\begin{array}{rlrl}
E & =1.08 \times 10^{5} \mathrm{~J} \mathrm{~mol}^{-1} & & \text { activation energy of dry reaction } \\
E_{w} & =6.5 \times 10^{4} \mathrm{~J} \mathrm{~mol}^{-1} & & \text { activation energy of wet reaction } \\
R & =8.31 \mathrm{~J} \mathrm{~mol}^{-1} \mathrm{~K}^{-1} & & \text { universal gas constant } \\
Q & =1.7 \times 10^{7} \mathrm{~J} \mathrm{~kg}^{-1} & & \text { exothermicity of dry reaction } \\
Z & =2.2 \times 10^{8} \mathrm{~s}^{-1} & & \text { pre-exponential of dry reaction } \\
Q_{w} \rho_{b} Z_{w} & =1.683 \times 10^{8} \mathrm{~J} \mathrm{~mol}^{-1} \mathrm{~s}^{-1} & \text { coefficient of wet reaction } \\
Z_{e} & =3.41 \times 10^{4} \mathrm{~s}^{-1} & & \text { pre-exponential of evaporation } \\
Z_{c} & =4.7 \mathrm{~s}^{-1} & & \text { pre-exponential of condensation } \\
L_{v} & =42 \times 10^{3} \mathrm{~J} \mathrm{~mol}^{-1} & & \text { latent heat of vaporization } \\
k & =0.5 \mathrm{~J} \mathrm{~m}^{-1} \mathrm{~K}^{-1} \mathrm{~s}^{-1} & & \text { thermal conductivity } \\
D & =2.5 \times 10^{-5} \mathrm{~m}^{2} \mathrm{~s}^{-1} & & \text { diffusitivity of vapour } \\
h & =5 \mathrm{~J} \mathrm{~m} \mathrm{~J}^{-1} \mathrm{~K}^{-1} & & \text { heat transfer coefficient } \\
m_{w} & =18 \times 10^{-3} \mathrm{~kg} \mathrm{~mol}^{-1} & & \text { mass of water } \\
\rho_{w} & =1 \times 10^{3} \mathrm{~kg} \mathrm{~m}^{-3} & & \text { density of water } \\
\rho_{b} & =125 \mathrm{~kg} \mathrm{~m}^{-3} & & \text { density of dry bagasse } \\
c_{b} & =1.34 \times 10^{3} \mathrm{~J} \mathrm{~kg}^{-1} \mathrm{~K}^{-1} & & \text { heat capacity of dry bagasse } \\
c_{w} & =4.19 \times 10^{3} \mathrm{~J} \mathrm{~kg}^{-1} \mathrm{~K}^{-1} & & \text { heat capacity of water }
\end{array}
$$

\section{References}

[1] T. Dixon, "Spontaneous combustion in bagasse stockpiles", Proc. Aust. Sugar Cane Tech. 10 (1988) 53-61.

[2] B. F. Gray, J. F. Griffiths, G. A. Foulds, B. G. Charlton and G. S. Walker, "The relevance of thermokinetic interactions and numerical model to the homogeneous partial oxidation of methane", Ind. Eng. Chem. Res. 33 (1994) 1126-1135. 
[3] B. F. Gray, J. F. Griffiths and S. M. Hasko, "Spontaneous ignition hazards in stockpiles of cellulosic materials: criteria for safe storage", J. Chem. Tech. Biotechnol. 34A (1984) 453-463.

[4] B. F. Gray and G. C. Wake, "The ignition of hygroscopic combustible materials by water", Combustion and Flame 79 (1990) 2-6.

[5] J. F. Griffiths and S. K. Scott, "Thermokinetic interactions: fundamentals of spontaneous ignition and cool flames", Prog. Energy and Combust. Sci. 13 (1987) 161-197.

[6] B. Halliburton, Ph. D. Thesis, School of Chemistry, Macquarie University, in preparation.

[7] D. W. Jordan and P. Smith, Nonlinear ordinary differential equations, 2nd ed. (Clarendon Press, Oxford, 1987).

[8] R. A. Sisson, A. Swift, G. C. Wake and B. F. Gray, "The self-heating of damp cellulosic materials. I. High thermal conductivity and diffusivity", IMA J. App. Math. 49 (1992) 273-29l.

[9] R. A. Sisson, A. Swift, G. C. Wake and B. F. Gray, "The self-heating of damp cellulosic materials. II. On the steady states of the spatially distributed case", IMA J. Appl. Math. 50 (1993) 285-306. 